\title{
Sodium-Glucose Cotransporter 2 Inhibitors and Major COVID-19 Outcomes: Promising Mechanisms, Conflicting Data, and Intriguing Clinical Decisions
}

\author{
Dimitrios Patoulias · Christodoulos Papadopoulos · Alexandra Katsimardou • \\ Maria Toumpourleka · Michael Doumas
}

Received: September 10, 2020 / Accepted: October 3, 2020 / Published online: October 14, 2020

(C) The Author(s) 2020

Keywords: Diabetes mellitus; Pandemic; SGLT2 inhibitors; SARS-CoV-2

\section{Key Summary Points}

SGLT2 inhibitors seem to be a promising adjunct treatment option in patients with SARS-CoV-2 infection and co-morbidities, based on pathophysiologic mechanisms.

A major concern that arises is the increased risk of protracted ketonemia and diabetic ketoacidosis that are difficult to resolve.

Current evidence does not support the use of SGLT2 inhibitors in infected patients.

D. Patoulias $(\bowtie) \cdot$ A. Katsimardou · M. Doumas Second Propedeutic Department of Internal Medicine, General Hospital "Hippokration", Aristotle University of Thessaloniki, Thessaloniki, Greece

e-mail: dipatoulias@gmail.com

C. Papadopoulos - M. Toumpourleka Third Department of Cardiology, General Hospital "Hippokration", Aristotle University of

Thessaloniki, Thessaloniki, Greece

M. Doumas

VAMC and George Washington University,

Washington, D.C., USA

\section{DIGITAL FEATURES}

To view digital features for this article go to https://doi.org/10.6084/m9.figshare.13041794.

Dear Editor,

In their anecdotal report, Bossi and colleagues demonstrate that "off-label" use of sodium-glucose cotransporter 2 (SGLT2) inhibitors in three subjects with severe or critical severe acute respiratory syndrome coronavirus 2 (SARS-CoV-2) pneumonia without diabetes did not have a significant impact on surrogate clinical outcomes, such as length of hospital stay [1]. In another recent retrospective analysis, Dalan et al. provide significant evidence regarding the impact of different antihypertensive and antihyperglycemic drug classes on coronavirus disease 2019 (COVID-19) "hard" outcomes [2]. Of note, the researchers demonstrated that among infected subjects with concomitant type 2 diabetes mellitus (T2DM), those prior treated with SGLT2 inhibitors $(n=16)$ featured a significantly lower risk of mechanical ventilation, after adjustment for baseline characteristics and other established cardiovascular risk factors [2]. The latter might have significant prognostic implications, based on the high mortality rates observed among patients that develop acute respiratory distress syndrome (ARDS) and are managed with mechanical ventilation [3]. 
Beyond their glucose-lowering effect, SGLT2 inhibitors exert pleiotropic effects (which are summarized by Bossi et al.), through which they provide substantial benefits in T2DM co-morbidities, such as cardiovascular disease, heart failure, and chronic kidney disease, all of which are associated with augmented risk of mortality from COVID-19 [4], while it is established that they confer cardiovascular benefit even in subjects without diabetes. The anti-inflammatory properties of SGLT2 inhibitors, mainly proven in experimental studies, might also be beneficial for patients with COVID-19, via amelioration of the so-called cytokine storm [5], despite the fact that its pathophysiologic significance has been recently doubted [6].

The major issue that arises against the use of SGLT2 inhibitors in patients with COVID-19 is the potentially increased risk of diabetic ketoacidosis (DKA) in the context of an acute infection $[7,8]$. Protracted ketonemia, delay in DKA resolution, and high mortality among patients with COVID-19 and DKA pose significant concerns regarding the use of SGLT2 inhibitors in the acute setting, despite the fact that the rates of SGLT2 inhibitor usage in the aforementioned series were low $[9,10]$.

In conclusion, current evidence seems insufficient to influence decision-making for the management of patients in the acute setting, especially those without diabetes. An individualized treatment approach is advisable. Until then, we have to wait for the results of the Dapagliflozin in Respiratory Failure in Patients with COVID-19 (DARE-19) trial (ClinicalTrials.gov identifier NCT04350593) and larger observational studies, to elucidate the exact role of this drug class in the COVID-19 pandemic.

\section{ACKNOWLEDGEMENTS}

Funding. No funding or sponsorship was received for this study or publication of this article.

Authorship. All named authors meet the International Committee of Medical Journal Editors (ICMJE) criteria for authorship for this article, take responsibility for the integrity of the work as a whole, and have given their approval for this version to be published.

Disclosure. Dimitrios Patoulias, Christodoulos Papadopoulos, Alexandra Katsimardou, Maria Toumpourleka and Michael Doumas have nothing to disclose.

Compliance with Ethics Guidelines. This article is based on previously conducted studies and does not contain any studies with human participants or animals performed by any of the authors.

Data Availability. Data sharing is not applicable to this article as no datasets were generated or analyzed during the current study.

Open Access. This article is licensed under a Creative Commons Attribution-NonCommercial 4.0 International License, which permits any non-commercial use, sharing, adaptation, distribution and reproduction in any medium or format, as long as you give appropriate credit to the original author(s) and the source, provide a link to the Creative Commons licence, and indicate if changes were made. The images or other third party material in this article are included in the article's Creative Commons licence, unless indicated otherwise in a credit line to the material. If material is not included in the article's Creative Commons licence and your intended use is not permitted by statutory regulation or exceeds the permitted use, you will need to obtain permission directly from the copyright holder. To view a copy of this licence, visit http:// creativecommons.org/licenses/by-nc/4.0/.

\section{REFERENCES}

1. Bossi AC, Forloni F, Colombelli PL. Lack of efficacy of SGLT2-i in severe pneumonia related to novel coronavirus (nCoV) infection: no little help from our friends. Diabetes Ther. 2020;11:1605-6.

2. Dalan R, Ang LW, Tan WYT, et al. The association of hypertension and diabetes pharmacotherapy with COVID-19 severity and immune signatures: an 
observational study. Eur Heart J Cardiovasc Pharmacother. 2020;2020:pvaa098. https://doi.org/10. 1093/ehjcvp/pvaa098.

3. Bellani G, Laffey JG, Pham T, et al. Epidemiology, patterns of care, and mortality for patients with acute respiratory distress syndrome in intensive care units in 50 countries. JAMA. 2016;315: 788-800.

4. Ssentongo P, Ssentongo AE, Heilbrunn ES, et al. Association of cardiovascular disease and 10 other pre-existing comorbidities with COVID-19 mortality: a systematic review and meta-analysis. PLoS One. 2020;15:e0238215.

5. Cowie MR, Fisher M. SGLT2 inhibitors: mechanisms of cardiovascular benefit beyond glycaemic control. Nat Rev Cardiol. 2020.

6. Kox M, Waalders NJB, Kooistra EJ, et al. Cytokine levels in critically ill patients with COVID-19 and other conditions. JAMA. 2020. https://doi.org/10. 1001/jama.2020.17052.

7. Scheen AJ. SGLT2 inhibition during the COVID-19 epidemic: friend or foe? Diabetes Metab. 2020. https://doi.org/10.1016/j.diabet.2020.06.003.

8. Das L, Dutta P. SGLT2 inhibition and COVID-19: the road not taken. Eur J Clin Invest. 2020;2020: e13339. https://doi.org/10.1111/eci.13339.

9. Armeni E, Aziz U, Qamar S, et al. Protracted ketonaemia in hyperglycaemic emergencies in COVID19: a retrospective case series. Lancet Diabetes Endocrinol. 2020;8:660-3.

10. Chamorro-Pareja N, Parthasarathy S, Annam J, et al. Letter to the editor: unexpected high mortality in COVID-19 and diabetic ketoacidosis. Metabolism. 2020;110:154301. 